\title{
ON ARITHMETIC FUNCTIONS AND DIVISORS OF HIGHER ORDER
}

\author{
KRISHNASWAMI ALLADI
}

(Received 15 January 1975)

\begin{abstract}
We discuss properties of arithmetic functions of higher order defined through the introduction of a new concept of divisor of higher order. We shall construct an infinite sequence of Euler-like functions and the well known Euler function will be the first member of this sequence. Asymptotic estimates of such functions are given and a study of error functions associated with the Euler-like sequence is made. We would like to mention that the familiar number theoretic functions become only the first members of an infinite sequence of functions of similar behaviour.
\end{abstract}

\section{Introduction}

If $d$ and $n$ are two positive integers and if $d \mid n$ we say $d$ is a first order divisor of $n$ and change the notation to $d \mid{ }_{1} n$. When $a$ and $b$ are two positive integers, $(a, b)$ rewritten as $(a, b)$, shall denote the largest divisor of $a$ dividing $b$. When $(a, b)_{1}=1$ we say $a$ is prime to $b$ first order.

If $d$ and $n$ are two positive integers then $d$ is said to be a divisor of $n$ of second order, denoted by $\left.d\right|_{2} n$ if

$$
\left(\frac{n}{d}, d\right)_{1}=1
$$

(This is the definition of unitary divisor). The symbol $(a, b)_{2}$ represents the largest divisor $c$ of $a$ satisfying $\left.c\right|_{2} b$. If $(a, b)_{2}=1$ we say $a$ is prime to $b$ order 2. Here comes the departure: A divisor $d$ of $n$ is a divisor of third order (notation: $\left.d\right|_{3} n$ ) if

$$
\left(\frac{n}{d}, d\right)_{2}=1 .
$$

The symbol $(a, b)_{3}$ stands for the largest divisor $c$ of $a$ that satisfies $\left.c\right|_{3} b$. If $(a, b)_{3}=1$ we say $a$ is prime to $b$ order 3 . We generalise by saying that $\left.d\right|_{r} n$ (read $d$ is $r$ th-order divisor of $n$ ) if 


$$
\left(\frac{n}{d}, d\right)_{r-1}=1
$$

and

$$
(a, b),=\max \left\{\left.c\right|_{1} a:\left.c\right|_{r} b\right\} .
$$

If $(a, b)_{r}=1$ then $a$ is prime to $b$ order $r$.

Note. The definition $\left.d\right|_{3} n$ given by us differs from the two well known extensions of the concept of unitary divisor given by Chidambaraswamy (1967) and Suryanarayana (1971) respectively. The former defines $d$ to be a semi-unitary divisor of $n$ if $(d, n / d)_{2}=1$ as opposed to our $\left.d\right|_{3} n$ where $(n / d, d)_{2}=1$. The latter defines $d$ to be a bi-unitary divisor of $n$ if $(d, n / d)^{* *}=1$ where $(a, b)^{* *}$ represents the largest common unitary divisor of $a$ and $b$. However in both these papers the concept of unitary divisor is just extended one step beyond.

Our definition of higher order divisors is given in such a way that the higher order divisors share many properties in common so that it is possible to discuss together the properties of arithmetic functions of $r$ th order, as we shall see in the theorems that follow. In fact to discuss the entire system as a unit, it becomes necessary to place $n / d$ on the left side. Moreover the familiar number theoretic results follow as corollaries if we set $r=1$, and some of the results of Cohen (1960) can be deduced if we set $r=2$.

We now define $r$ th order analogues to some well known arithmetic functions. However as $(a, b)_{r} \neq(b, a)$, in general these functions have interesting dual functions. Denote by

$$
\varphi_{r}(n, x)=\sum_{\substack{n<a \leq x \\(a, n)_{r}=1}} 1 ; \quad \varphi_{r}(n, n)=\varphi_{r}(n)
$$

and its dual

$$
\varphi_{r}^{*}(n, x)=\sum_{\substack{0<a \leq x \\(n, a)_{r}=1}} 1 ; \quad \varphi_{r}^{*}(n, n)=\varphi_{r}^{*}(n)
$$

for $r \geqq 1$. We define $\varphi_{0}(n, x)=\varphi_{0}^{*}(n, x)=[x]$ where $[x]$ denotes the largest integer $\leqq x$ and $(a, n)_{0}=(n, a)_{0}=1$ for all $a$ and $n$. Note that $\varphi_{1}=\varphi_{1}^{*}=\varphi$ (Euler). We define the divisor functions

$$
\sigma_{r, k}(n)=\sum_{\left.d\right|_{r}, n} d^{k} \quad \text { and } \quad \sigma_{r, k}^{*}(n)=\sum_{d \mid, n}\left(\frac{n}{d}\right)^{k}
$$

and

$$
\sigma_{r, 0}(n)=\sigma_{r .0}^{*}(n)=\tau_{r}(n) .
$$


Before we take up the study of these functions we need to define some more functions. Let $\left\{F_{r}\right\}_{r=0}^{x}$ denote the sequence given by

$$
F_{0}=0, F_{1}=1, F_{n}=F_{n-1}+F_{n-2}, \quad n \geqq 2 .
$$

Let $l(y)$ and $l^{*}(y)$ denote respectively the least integer $>$ and $\geqq y$. Further define

$$
\begin{aligned}
& f_{r}(x)=l\left(\frac{F_{r-1}}{F_{r}} x\right) \text { when } r \equiv 1(\bmod 2) \\
& f_{r}(x) \equiv l^{*}\left(\frac{F_{r-1}}{F_{r}} x\right) \text { when } \quad r \equiv 0(\bmod 2) .
\end{aligned}
$$

Let $f_{r}^{-1}(x)$ denote the largest integer $y$ with $f_{r}(y)=x$. And if $n=\Pi_{i=1}^{s} p_{i}^{\alpha_{i}}$ be the canonical decomposition of $n$ then let

$$
\beta_{r}(n)=\prod_{i=1}^{5} p_{i^{\prime}}^{f^{-1}\left(\alpha_{i}\right)+1} r>1, \quad \beta_{1}(n)=n .
$$

\section{Properties of higher order divisors and arithmetic functions}

We will now show

THEOREM 1. If $n=\prod_{i=1}^{s} p_{i}^{\alpha_{i}}$ be the canonical representation of $n$ as a product of distinct primes, and if $\left.d\right|_{1} n$ then $\left.d\right|_{,} n$ if and only if $d=\Pi_{i=1}^{s} p_{i}^{\beta_{i}}$ where

$$
\beta_{i}=0 \quad \text { or } \quad f_{r}\left(\alpha_{i}\right) \leqq \beta_{i} \leqq \alpha_{i} \quad i=1,2, \cdots, s .
$$

PRoOF. For $r=1$ by (1.9) and (1.10) $f_{r}\left(\alpha_{i}\right)=1$ and so the theorem holds trivially. For $r=2, f_{r}\left(\alpha_{i}\right)=\alpha_{i}$ (again by (1.9) and (1.11)) and $\beta_{i}=0$ or $\beta_{i}=\alpha_{i}$ for a unitary divisor and the theorem is true.

Let $r=3$ and $d=\prod_{i=1}^{s} p_{i}^{\beta_{i}}$ satisfy $\left.d\right|_{3} n$. Clearly $\left.d\right|_{1} n$ and so $\alpha_{i} \geqq \beta_{i}$ trivially holds. Now

$$
\frac{n}{d}=\prod_{i=1}^{s} p_{i}^{\alpha_{i}-\beta_{i}}
$$

If $\left.d\right|_{3} n$ then $(n / d, d)_{2}=1$. Thus there is no divisor of $n / d$ except 1 which is a divisor of $d$ of second order. This is possible if and only if

$$
\alpha_{i}-\beta_{i}<\beta_{i} \text { or } \beta_{i}=0 .
$$

For if $\alpha_{i}-\beta_{i} \geqq \beta_{i}$ then $\left.p_{i}^{\beta_{i}}\right|_{1} n / d$ and $\left.p_{i}^{\beta_{1}}\right|_{2} d$ and this is a contradiction. Thus $\alpha_{i}-\beta_{i}<\beta_{i}$. If $\alpha_{i}-\beta_{i}<\beta_{i}$, and $p^{\nu_{i}} \mid n / d$ then $0 \leqq \nu_{i} \leqq \alpha_{i}-\beta_{i}<\beta_{i}$ so that $\left.p_{i}^{\nu_{i}}\right|_{2} d$. Hence $(n / d, d)_{2}=1$. Thus 


$$
\alpha_{i}-\beta_{i}<\beta_{i} \Leftrightarrow \beta_{i}>\frac{\alpha_{i}}{2}=\frac{F_{2}}{F_{3}} \alpha_{i}
$$

Moreover $\beta_{i}$ is an integer and so $\beta_{i} \geqq f_{3}\left(\alpha_{i}\right)$ proving theorem for $r=3$.

In general let the theorem hold for $1,2, \cdots, r, r$ even. Now $\left.d\right|_{r+1} n$ if and only if $(n / d, d)_{r}=1$ where $n$ and $d$ are represented as above. Now $(n / d, d)_{r}=$ 1 says that there is no divisor of $n / d$ save 1 that is a divisor of $d$ order $r$. This is possible if and only if

$$
\alpha_{i}-\beta_{i}<\frac{F_{r-1}}{F_{r}} \beta_{i} \text { or } \beta_{i}=0 .
$$

For otherwise if $\alpha_{i}-\beta_{i} \geqq F_{r-1} F_{r} / \beta_{i}$ then one can find a $\nu_{i}$ satisfying

$$
\alpha_{i}-\beta_{i} \geqq \nu_{i} \geqq F_{r-1} \beta_{i} / F_{r}
$$

so that $\left.p^{v_{i}}\right|_{1}(n / d)$ and $p_{i}^{\nu_{i}} \mid, d$, a contradiction. Thus (2.1) holds. The sufficiency of (2.1) is clear. We rewrite $(2.1)$ as

$$
\beta_{i}>\frac{F_{r}}{F_{r+1}} \alpha_{i}
$$

and $\beta_{i}$ is an integer. Thus $\beta_{i} \geqq f_{r+1}\left(\alpha_{i}\right)$ proving lemma for $r+1$ odd. The proof for the case $r+1$ even is similar, only that $\beta_{i} \geqq F_{r} \alpha_{i} / F_{r+1}$ will replace (2.2) for (2.1) will be replaced by a weak inequality.

The higher order divisors share in common the property

THEOREM 2. (a) If $a$ and $n$ are integers, then for any nonnegative integer $\lambda$

$$
(a, n)_{r}=(\lambda n+a, n)_{r}=(\lambda n-a, n)_{r} .
$$

(b) We have $(n, a)_{r}=1$ if and only if

$$
(n, a)_{r}=\left(n, \lambda \beta_{r}(n)+a\right)_{r}=\left(n, \lambda \beta_{r}(n)-a\right)_{r}=1
$$

where $\beta_{r}(n)$ is as defined in (1.12).

We omit the proofs of Theorem 2 as they are direct consequences of the definitions in section 1 . We shall need Theorem 2 in the discussion of error functions.

We now take up the study of the functions defined in (1.5) to (1.8). We shall always represent $n$ in the canonical form $n=\Pi_{i=1}^{s} p_{i}^{\alpha_{i}}$.

ThEOREM 3. $\varphi_{r}(n)=n \prod_{i=1}^{s}\left(1-\frac{1}{p_{i}^{f_{(}^{\left(\alpha_{i}\right)}}}\right)$ for $r \geqq 1$.

Proof. We know from (1.5) that 


$$
\varphi_{r}(n, x)=\sum_{\substack{0<a \leq x \\(a, n)_{r}=1}} 1=[x]-\sum_{\substack{0<a \leq x \\(a, n)_{r}>1}} 1 .
$$

Now $(a, n)_{r}>1$ if there exists $\left.d\right|_{r} n, d>1$ with $\left.d\right|_{1} a$. We know from Theorem 1 that $\left.d\right|_{r} n$ if and only if $\beta_{i}=0$ or $f_{r}\left(\alpha_{i}\right) \leqq \beta_{i} \leqq \alpha_{i}$. This implies that if $p_{i} \mid a$ and $p_{i} \mid{ }_{r} n$ then $\left.p_{i}^{f^{\prime}\left(\alpha_{i}\right)}\right|_{1} a$. Thus a simple combinatorial argument leads to

$$
\varphi_{r}(n, x)=[x]-\sum_{0<i \leqq s}\left[\frac{x}{p_{i}^{f_{i}\left(\alpha_{i}\right)}}\right]+\sum_{0<i<j \leqq s}\left[\frac{x}{p_{i}^{f_{i}^{(}\left(\alpha_{i}\right)} p_{i}^{f_{f}\left(\alpha_{j}\right)}}\right]
$$

$$
-\cdots+(-1)^{s} \sum\left[\frac{x}{p_{1}^{f_{(}^{(}\left(\alpha_{1}\right)} \cdots p_{s}^{f_{(}^{\prime}\left(\alpha_{s}\right)}}\right] .
$$

If we put $x=n$ in (2.5) we get Theorem 3 .

Now (2.5) also indicates that

CoRollaRy 1. If $e_{r}(n, x)=x \varphi_{r}(n) / n-\varphi_{r}(n, x)$ then

$$
e_{r}(n, x)=0\left(\tau_{1}(n)\right)
$$

Proof. We can rewrite $(2.5)$ as

$$
\begin{aligned}
\varphi_{r}(n, x) & =x-\sum \frac{x}{p_{i}^{f^{\prime}\left(\alpha_{i}\right)}}+\cdots+0\left(1+\sum_{p_{i} \mid n} 1+\sum_{p_{i} p_{j} \mid n}+\cdots\right) \\
& =\frac{x \varphi_{r}(n)}{n}+0\left(\tau_{1}(n)\right) .
\end{aligned}
$$

THEOREM 4. With $\beta,(n)$ as in (1.12) we have

$$
\varphi_{r}^{*}\left(n, \beta_{r}(n)\right)=\varphi_{1}\left(n, \beta_{r}(n)\right) \prod_{i=1}^{s}\left(1+\frac{1}{p_{i}^{f^{\prime, 1}\left(\alpha_{i}\right)+1}\left(1-\frac{1}{p_{i}}\right)}\right)
$$

Proof. Going back to the definition of $\varphi_{r}^{*}(n, x)$ in (1.6) we find that $(n, a)_{r}=1$ can arise out of two cases. If $(n, a)_{1}=1$ then $(n, a)_{r}=(a, n)_{r}=1$. Or $(n, a)_{1}>1$ in which case there is a $\left.p_{i}\right|_{1} n$ and $\left.p_{i}\right|_{1} a$. As $(n, a)_{r}=1$ even if $d_{1} \mid a$, $d \nmid, n$ for all $d \mid, n$. Thus $\left.p_{i}^{f-1\left(\alpha_{i}\right)+1}\right|_{1} a$. Again a simple combinatorial argument leads to

$$
\varphi_{r}^{*}(n, x)=\varphi_{1}(n, x)+\sum_{0<i \leqq s} \varphi_{1}\left(\frac{n}{p_{i}^{\alpha_{i}}}, \frac{x}{p_{i}^{f^{-1}}\left(\alpha_{i}\right)+1}\right)
$$

$$
+\sum_{0<i<j \leqq s} \varphi_{1}\left(\frac{n}{p_{i}^{\alpha} p_{j}^{\alpha_{j}}}, \frac{x}{p_{i}^{f_{i}^{-1}\left(\alpha_{j}\right)+1} p_{j}^{f_{i}^{-1}\left(\alpha_{j}\right)+1}}\right)+\cdots
$$

If we put $x=\beta_{r}(n)$ in (2.6) and use Theorem 2 which for $r=1$ gives $\varphi_{1}(n, \lambda n+\mu)=\lambda \varphi_{1}(n)+\varphi_{1}(n, \mu)$ we get Theorem 4 . 
CoROLlaRY 2. If $e_{r}^{*}(n, x)=x \varphi_{r}^{*}\left(n, \beta_{r}(n)\right) / \beta_{r}(n)-\varphi_{r}^{*}(n, x)$ then $e_{r}^{*}(n, x)=0\left(\tau_{1}(n)\right)$.

We omit details of the proof which is similar to Corollary 1.

Similarly formulae can be found for $\sigma_{r, k}(n), \sigma_{r, k}^{*}(n)$, and $\tau_{r}(n)$. These are given below

$$
\tau_{r}(n)=\prod_{i=1}^{s}\left(\alpha_{i}-f_{r}\left(\alpha_{i}\right)+2\right)
$$

and

$$
\sigma_{r, k}(n)=\prod_{i=1}^{s}\left(1+p_{i}^{f_{i}^{(}\left(\alpha_{i}\right)}+\cdots p_{i}^{\alpha_{i}}\right) .
$$

We are now in a position to prove the following which is somewhat interesting since it is novel.

THEOREM 5. For any pair of integers $n$ and $k \geqq 0$ we have

$$
\begin{aligned}
& \varphi_{1}(n) \leqq \varphi_{3}(n) \leqq \varphi_{5}(n) \leqq \cdots \leqq \varphi_{6}(n) \leqq \varphi_{4}(n) \leqq \varphi_{2}(n) \leqq \varphi_{0}(n) \\
& \text { (b) } \quad \sigma_{2, k}(n) \leqq \sigma_{4, k}(n) \leqq \sigma_{6, k}(n) \leqq \cdots \leqq \sigma_{5, k}(n) \leqq \sigma_{3, k}(n) \leqq \sigma_{1, k}(n) \\
& \text { (c) } \quad \sigma_{2, k}^{*}(n) \leqq \sigma_{4, k}^{*}(n) \leqq \sigma_{6, k}^{*}(n) \leqq \cdots \leqq \sigma_{S, k}^{*}(n) \leqq \sigma_{3, k}^{*}(n) \leqq \sigma_{1, k}^{*}(n) \\
& \frac{\varphi_{1}^{*}\left(n, \beta_{1}(n)\right)}{\beta_{1}(n)} \leqq \frac{\varphi_{3}^{*}\left(n, \beta_{3}(n)\right)}{\beta_{3}(n)} \leqq \frac{\varphi_{5}^{*}\left(n, \beta_{5}(n)\right)}{\beta_{5}(n)} \\
& \leqq \ldots \frac{\varphi_{6}^{*}\left(n, \beta_{6}(n)\right)}{\beta_{6}(n)} \leqq \frac{\varphi_{4}^{*}\left(n, \beta_{4}(n)\right)}{\beta_{4}(n)} \leqq \frac{\varphi_{2}^{*}\left(n, \beta_{2}(n)\right)}{\beta_{2}(n)} .
\end{aligned}
$$

Proof. We shall prove (a) and (b). The proofs of (c) and (d) are similar. First we observe that $F_{2 k} / F_{2 k+1}$ form an increasing sequence and $F_{2 k-1} / F_{2 k}$ form a decreasing sequence both sequences converging to $(\sqrt{5}-1) / 2$. Further if $x<y$ then

$$
l(x) \leqq l(y) \quad l^{*}(x) \leqq l^{*}(y) \text { and } \quad l(x) \leqq l^{*}(y) .
$$

These follow from the definitions of $l$ and $l^{*}$. Now (2.9) implies that for any integer $m$ we have

$$
f_{1}(m) \leqq f_{3}(m) \leqq f_{5}(m) \leqq \cdots \leqq f_{6}(m) \leqq f_{4}(m) \leqq f_{2}(m) .
$$

If we use (2.10) and Theorem 3 we get (a). Now (2.9) and (2.10) will give on similar lines of reasoning the reverse inequalities for $f_{r}^{-1}(m)$. Then if we use Theorem 4 we get (d).

To prove (b) and (c) it is enough to observe that (2.10) implies that

$$
\left.\left.d\right|_{2 m} n \Rightarrow d\right|_{2 m+2} n ;\left.\left.d\right|_{2 m+1} n \Rightarrow d\right|_{2 m-1} n ;\left.\left.d\right|_{2 m} n \Rightarrow d\right|_{2 m^{\prime}+1} n
$$


for any pair of integers $m$ and $m^{\prime}$. That is if $D_{m}(n)$ denotes the set of $m$ th order divisors of $n$ then

$$
D_{2}(n) \subset D_{4}(n) \subset D_{6}(n) \subset \cdots \subset D_{5}(n) \subset D_{3}(n) \subset D_{1}(n) .
$$

Clearly (2.11) gives (b) and (c). This proves the theorem.

\section{Asymptotic estimates}

We saw in the last section properties of $\left.d\right|_{r} n$. Theorem 5 gave for instance relations between $\varphi_{r}, \sigma_{r, k}, \varphi_{r}^{*}$ and $\sigma_{r, k}^{*}$ for $r=1,2, \cdots$ separately. When we take up asymptotic estimates of these functions, we find that the $\varphi$ 's and the $\sigma$ 's are related. For example the average order of $\sigma_{r, k}$ and $\sigma_{r, k}^{*}$ involve $\varphi_{r-1}^{*}$ and $\varphi_{r-1}$ respectively. We begin by proving

THEOREM 6. There, exists a constant $c_{r}$ so that

$$
\sum_{1 \leqq n \leq x} \varphi_{r}(n)=c_{r} x^{2}+0\left(x^{3 / 2+\varepsilon}\right) \forall \varepsilon>0 .
$$

Proof. We note that Theorem 3 implies that $\varphi$ is multiplicative. Also if $n$ is square free then $\varphi_{r}(n)=\varphi_{1}(n)$.

Decompose every number $n$ as $n=N n^{\prime}$ where $n^{\prime}$ is square free and $\left(n^{\prime}, N\right)=1$. The number $N$ has the property that if $p \mid N, p$-prime then $p^{2} \mid N$. ( $N$ is called a powerful number.) We call $N$ the powerful part of $n$. Keep $N$ fixed and ask for those $n \leqq x$ for which $N$ is the powerful part. Sum over all such $N$. Thus

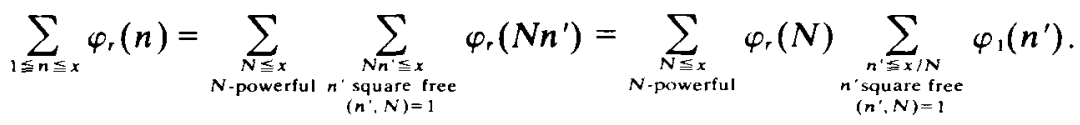

So we need to know the sum

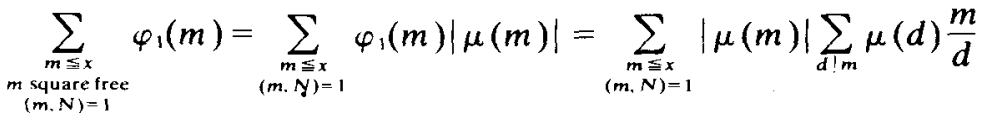

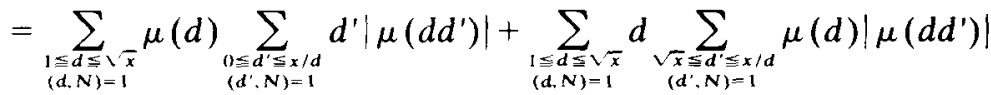

$$
\begin{aligned}
& =\sum_{\substack{1 \leq d \leq V_{x} \\
(d, N)=1}} \mu(d) \sum_{\substack{0 \leq d^{\prime} \leq x / d \\
\left(d^{\prime}, N\right)=1}} d^{\prime}\left|\mu\left(d d^{\prime}\right)\right|+0\left(x^{3 / 2}\right) \\
& =\sum_{\substack{1 \leq d \leq 5 / \bar{x} \\
(d, N)=1}} \mu(d) \sum_{\substack{d \leq x / d \\
d^{\prime} \text { 'square free } \\
\left(d^{\prime} \cdot N d\right)=1}} d^{\prime}+0\left(x^{3 / 2}\right)
\end{aligned}
$$


The function $\mu$ used above is the Möbius function. It is not difficult to show that the number of integers $\leqq x$ that are square free and prime to $N d$ is

$$
c_{N d} x+0(\sqrt{x})
$$

where

$$
c_{N d}=\prod_{p \mid N d}\left(1-\frac{1}{p}\right) \cdot \prod_{q+N d}\left(1-\frac{1}{q^{2}}\right)=\frac{6}{\pi^{2}} \prod_{p \mid N d}\left(1+\frac{1}{p}\right)^{-1}<\frac{6}{\pi^{2}} .
$$

So the sum in (3.2) is of the form

$$
\frac{x^{2}}{2} \sum_{\substack{1 \leq d \leq \sqrt{x} \\(d, N)=1}} \frac{\mu(d)}{d^{2}} c_{N d}+0\left(x^{3 / 2}\right)=k_{N} \frac{x^{2}}{2}+0\left(x^{3 / 2}\right)
$$

where $k_{N}$ is a constant depending on $N$ and bounded for all $N$-powerful. Substituting this in (3.1) we get

$$
\sum_{1 \leq n \leq x} \varphi_{r}(n)=x^{2} \sum_{\substack{N \leq x \\ N \text { powerful }}} k_{N} \frac{\varphi_{r}(N)}{2 N^{2}}+0\left(x^{3 / 2}\right) .
$$

Now every powerful number $N$ is of the form $S S^{\prime}$ where $S$ is a perfect square, $S^{\prime}$ square free and $S^{\prime} \mid S$. This decomposition is unique. So the number of powerful numbers $\leqq x$ is

$$
0\left(\sum_{\substack{S \leq x \\ S \text { square }}} \tau_{1}(S)\right)=0\left(x^{1 / 2+\varepsilon}\right) \forall \varepsilon>0 .
$$

This shows that the series

$$
\sum_{\substack{N=1 \\ N \text {. powerful }}}^{\infty} \frac{\varphi_{r}(N) k_{N}}{2 N^{2}}<\infty
$$

so that if $c_{r}$ is the sum of this series we have

$$
\sum_{1 \leq n \leq x} \varphi_{r}(n)=c_{r} x^{2}+0\left(x^{3 / 2+\varepsilon}\right) \forall \varepsilon>0
$$

which proves the theorem.

It is interesting to observe

THEOREM 7. We have

$$
\sum_{1 \leq n \leq x} \frac{\varphi_{r}(n)}{n} \sim \sum_{1 \leq n \leq x} \frac{\varphi_{r}^{*}\left(n, \beta_{r}(n)\right)}{\beta_{r}(n)} \sim 2 c_{r} x .
$$

Proof. We begin by stating Abel's summation formula as in LeVeque (1960). 
"Suppose that $\lambda_{1}, \lambda_{2}, \cdots$ is a non-decreasing sequence with limit infinity, that $a_{1}, a_{2}, \cdots$ is an arbitrary sequence of real or complex numbers, and $f$ a function with a continuous derivative for $x \geqq \lambda_{1}$. Put

$$
A(x)=\sum_{\lambda_{n} \leq x} a_{n} .
$$

Then for $x \geqq \lambda_{1}$

$$
\sum_{\lambda_{n} \leqq x} a_{n} f\left(\lambda_{n}\right)=A(x) f(x)-\int_{\lambda_{1}}^{x} A(t) f^{\prime}(t) d t . "
$$

Clearly, by Theorem 6 and Abel's summation formula with $\lambda_{n}=n$, $f(x)=1 / x$ and $a_{n}=\varphi_{r}(n)$ we infer that

$$
\sum_{1 \leq n \leqq x} \frac{\varphi_{r}(n)}{n}=2 c_{r} x+0\left(x^{\frac{1}{2+\epsilon}}\right) \forall \varepsilon>0
$$

which proves half the assertion. The second half is more interesting. We have

$$
\begin{aligned}
\sum_{n=1}^{m} \varphi_{r}(n, m) & =\sum_{n=1}^{m} \sum_{\substack{1 \leq a \leq m \\
(a, n)_{r}=1}} 1=\sum_{1 \leq a \leq m} \sum_{\substack{n=1 \\
(a, n)_{r}=1}}^{m} 1 \\
& =\sum_{a=1}^{m} \varphi_{*}^{*}(a, m)=\sum_{n=1}^{m} \varphi_{r}^{*}(n, m) .
\end{aligned}
$$

But

$$
\sum_{n=1}^{m} \varphi_{r}(n, m)=m \sum_{n=1}^{m} \frac{\varphi_{r}(n)}{n}+0(m \log m)
$$

by virtue of Corollary 1 and

$$
\sum_{n=1}^{m} \varphi_{r}^{*}(n, m)=m \sum_{n=1}^{m} \frac{\varphi_{r}^{*}\left(n, \beta_{r}(n)\right)}{\beta_{r}(n)}+0(m \log m)
$$

by Corollary 2 for

$$
\sum_{n \leq m} \tau_{1}(n)=0(m \log m)
$$

Replacing $m$ by $x$, dividing by $x$ we see that (3.4), (3.5) and (3.6) give Theorem 7.

We can now prove

THEOREM 8. $\quad \sum_{1 \leqq n \leqq m} \tau_{r}(n)=2 c_{r-1} m \log m+0(m)$.

Proof. Let us first give an interpretation to the sum 


$$
\sum_{1 \leq n \leq m} \tau_{r}(n)=\sum_{1 \leqq n \leqq m} \sum_{\left.d\right|_{r} n} 1=\sum_{1 \leqq n \leqq m} \sum_{(n / d, d)_{r-1}=1} 1 .
$$

So (3.7) represents the number of lattice points $\left(x_{0}, y_{0}\right)$ under the graph of the hyperbola $x y=m, x>0, y>0$ with $\left(y_{0}, x_{0}\right)_{r-1}=1$. We first count the lattice points with $x_{0} \leqq \sqrt{m}$. They are given by

$$
\sum_{1 \leq d \leqq \sqrt{m}} \sum_{\substack{d \\\left(d^{\prime}, d\right)_{r-1}=1}} 1=\sum_{1 \leqq d \leqq \sqrt{m}} \varphi_{r-1}(d, m / d)
$$

$$
\begin{aligned}
& =\sum_{d \leqq \sqrt{m}}\left\{m \frac{\varphi_{r-1}(d)}{d^{2}}+0\left(\tau_{1}(d)\right)\right\} \\
& =m \sum_{d \leq \sqrt{m}} \frac{\varphi_{r-1}(d)}{d^{2}}+O(\sqrt{m} \log m) .
\end{aligned}
$$

Now again Abel's summation formula and Theorem 7 tell us that the summation in (3.8) is

$$
2 c_{r-1} m \log \sqrt{m}+0(\sqrt{m} \log m) .
$$

Now the number of lattice points with $y_{0} \leqq \sqrt{m}$ is

$$
\sum_{1 \leqq d \leqq \sqrt{m}} \sum_{\substack{d \leq m^{\prime} / d \\\left(d, d^{\prime}\right)_{r-1}=1}} 1=\sum_{1 \leqq d \leqq \sqrt{m}} \varphi_{r-1}^{*}(d, m / d)
$$

which by use of Corollary 2 is

$$
m \sum_{d \leq \sqrt{m}} \frac{\varphi_{r-1}^{*}\left(d, \beta_{r-1}(d)\right)}{d \beta_{r-1}(d)}+0(\sqrt{m} \log m) .
$$

Again the use of Abel's summation formula and Theorem (7) gives that this sum is

$$
2 c_{r-1} m \log \sqrt{m}+0(\sqrt{m} \log m) .
$$

The overlap in these two processes of counting is the points in the square $1 \leqq x_{0} \leqq \sqrt{m}, 1 \leqq y_{0} \leqq \sqrt{m}$, which is $0(m)$. Thus

$$
\begin{aligned}
\sum_{n \leqq m} \tau_{r}(n) & =2 c_{r-1} m \log \sqrt{m}+2 c_{r-1} m \log \sqrt{m}+0(\sqrt{m} \log m)+0(m) \\
& =2 c_{r-1} m \log m+0(m) .
\end{aligned}
$$

COROLlaRY 3. If $\tau_{1}(n)$ is the divisor function then

$$
\sum_{n \leq m} \tau_{1}(n) \sim m \log m
$$


Proof. $\quad c_{0}=1 / 2$. Conclusion is clear.

COROLlaRY 4. If $\tau_{2}(n)$ is the number of unitary divisors of $n$ then

$$
\sum_{n \leqq m} \tau_{2}(n) \sim \frac{6}{\pi^{2}} m \log m .
$$

Proof. By Theorem 6, the constant $c_{1}=3 / \pi^{2}$. So $2 c_{1}=6 / \pi^{2}$ and corollary holds.

REMARK. The average order of $\varphi_{r}(n)$ is $2 c_{r} n$, while the average order of $\tau_{r}(n)$ is $2 c_{r-1} \log n$.

We now take up the asymptotic estimates of $\sigma_{r, k}$ and $\sigma_{r, k}^{*}$ for $k>0$. For $k>0$ define two constants

$$
\alpha_{r, k}=\frac{1}{k+1} \sum_{n=1}^{\infty} \frac{\varphi_{r-1}(n)}{n^{k+2}}
$$

and

$$
\alpha_{r, k}^{*}=\frac{1}{k+1} \sum_{n=1}^{\infty} \frac{\varphi_{r-1}^{*}\left(n, \beta_{r-1}(n)\right)}{\beta_{r-1}(n) \cdot n^{k+1}} .
$$

Our theorem is

THEOREM 9.

$$
\begin{aligned}
& \text { (a) } \sum_{n=1}^{m} \sigma_{r, k}(n)=\alpha_{r, k}^{*} m^{k+1}+0\left(m^{k+\frac{1}{2}}\right) ; \\
& \text { (b) } \sum_{n=1}^{m} \sigma_{r, k}^{*}(n)=\alpha_{r, k} m^{k+1}+0\left(m^{k+\frac{1}{2}}\right) .
\end{aligned}
$$

Proof. We shall prove the second part of the theorem. Part (a) will follow on similar reasoning. We shall first need an estimate of

$$
\sum_{0<a \leq x,(a, n)_{r}=1} a^{k} .
$$

Let $A(n, r, s)$ denote the $s$ th number ' $a$ ' such that $(a, n)_{r}=1$. It is obvious that

$$
\varphi \cdot(n, A(n, r, s))=s .
$$

But from Corollary 1 we infer that

$$
\varphi_{r}(n, A(n, r, s))=\frac{A(n, r, s)}{n} \varphi_{r}(n)+0\left(n^{\varepsilon}\right)=s
$$

so that 


$$
A(n, r, s)=\frac{n s}{\varphi_{r}(n)}+\frac{n}{\varphi_{r}(n)} \cdot 0\left(n^{\varepsilon}\right) \forall \varepsilon>0 .
$$

We deduce from Theorem 5 that for $r \geqq 0 \varphi_{r}(n) \geqq \varphi_{1}(n)=\varphi(n)$. Since it is known that $n / \varphi_{1}(n)=0(\log \log n)$ we infer that

$$
\frac{n}{\varphi_{r}(n)}=0(\log \log n)
$$

so that $(3: 12)$ is rewritten as

$$
A(n, r, s)=\frac{n s}{\varphi_{r}(n)}+0\left(n^{\varepsilon}\right) \forall>0 .
$$

Thus

$$
\begin{aligned}
\sum_{\substack{0<a \leqq x \\
(a, n)_{r}=1}} a^{k} & =\sum_{0<s \leqq \varphi_{r}(n, x)} A(n, r, s)^{k}=\sum_{0<s \leqq \varphi_{r}(n, x)}\left(\frac{n s}{\varphi_{r}(n)}+0\left(n^{\varepsilon}\right)\right)^{k} \forall \varepsilon>0 \\
& =\left\{\sum_{0<s \leqq \varphi_{r}(n, x)} s^{k}\right\} \frac{n^{k}}{\varphi_{r}(n)^{k}}+\frac{n^{k-1}}{\varphi_{r}(n)^{k-1}} \sum_{0<s \leqq \varphi_{r}(n, x)} 0\left(s^{k-1} n^{\varepsilon}\right) \forall \varepsilon>0 \\
& =\frac{n^{k}}{\varphi_{r}(n)^{k}}\left(\frac{\varphi_{r}}{k+1} \frac{(n)^{k+1}}{k+1}+0\left(\varphi_{r}(n, x)^{k}\right)\right)+0\left(\frac{n^{k-1+\varepsilon}}{\varphi_{r}(n)^{k-1}} \varphi_{r}(n, x)^{k}\right) \\
& =\frac{n^{k}}{\varphi_{r}(n)^{k}}\left(\frac{x^{k+1} \varphi_{r}(n)^{k+1}}{(k+1) n^{k+1}}+0\left(x^{k+\varepsilon}\right)\right)+0\left(n^{\varepsilon} \varphi_{r}(n, x)^{k}\right) \\
& =\frac{x^{k+1} \varphi_{r}(n)}{(k+1) n}+0\left(x^{k+e}\right) \forall \varepsilon>0
\end{aligned}
$$

where $x$ is taken as $\geqq n$.

We shall return to (3.14) after making a geometric interpretation of $\sum \sigma_{r, k}^{*}$. Consider the lattice points discussed in Theorem 8 . Call these lattice points 'good' and let $G$ denote the set of good lattice points. Divide the region under the curve into three non-intersecting regions $A, O P$, and $B$. Clearly

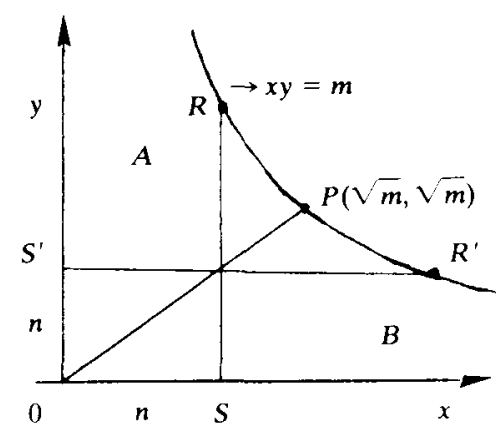




$$
\sum_{n=1}^{m} \sigma_{r, k}^{*}(n)=\sum_{\left(x_{0}, y_{0}\right) \in G} y_{0}^{k}
$$

which can be split up as

$$
\begin{aligned}
\sum_{n=1}^{m} \sigma_{r, k}^{*}(n) & =\sum_{\left(x_{1}, y_{0}\right) \in G \cap A} y_{0}^{k}+\sum_{\left(x_{0}, y_{0}\right) \in G \cap B} y_{0}^{k}+\sum_{\left(x_{0}, y_{0}\right) \in G \cap(O P)} y_{0}^{k} \\
& =S_{1}+S_{2}+S_{3} \text { say. }
\end{aligned}
$$

Clearly

$$
S_{3}=0\left(m^{(k+1) / 2}\right) .
$$

To estimate $S_{2}$, pick a point $S^{\prime}$ on $O Y$ at a distance $n$ from 0 with $n \leqq \sqrt{m}$. The sum of $y_{0}^{k}=n^{k}$ over $R^{\prime} S^{\prime}$ through $S^{\prime}$ is

$$
\sum_{n<x_{0} \leqq m / n:\left(n, x_{0}\right)_{r-1}=1} n^{k}=n^{k} \varphi_{r-1}^{*}(n, n, m / n)
$$

where

$$
\varphi_{r-1}^{*}(n, c, d)=\sum_{c<a \leqq d,(n, a)_{r-1}=1} 1
$$

Thus we have

$$
\begin{aligned}
S_{2} & =\sum_{n=1}^{[\sqrt{m}]} n^{k} \varphi_{r-1}^{*}(n, n, m / n)=\sum_{n=1}^{[\sqrt{m}]} n^{k} 0(m / n)=0\left(m^{(k+2) / 2}\right) \\
& =0\left(m^{k+1 / 2}\right) \quad \text { for } \quad k \geqq 1 .
\end{aligned}
$$

To estimate $S_{1}$ pick an $S$ on $O X$ and a distance $n$ from $0, n \leqq \sqrt{m}$. Draw $R S$ through it. The sum of $y_{0}^{k}$ over $y_{0}$ lying on $R S$ is

$$
\sum_{\substack{n<y_{0} \leq m / n \\\left(y_{0}, n\right)_{r-1}=1}} y_{0}^{k}=\frac{m^{k+1} \varphi_{r-1}(n)}{(k+1) n^{k+2}}+0\left(\frac{m^{k+\varepsilon}}{n^{k+\varepsilon}}\right)-\frac{n^{k} \varphi_{r-1}(n)}{k+1}+0\left(n^{k+\varepsilon}\right)
$$

using (3.14) where $x$ takes values $n$ and $m / n \geqq n$. If we sum from 1 to $\sqrt{m}$ we get $S_{1}$ which is

$$
\begin{aligned}
S_{1} & =\frac{m^{k+1}}{k+1} \sum_{n=1}^{(\sqrt{m})} \frac{\varphi_{r-1}(n)}{n^{k+2}}+0\left(m^{k+e}\right)+0\left(m^{(k+2) / 2}\right) \\
& =\frac{m^{k+1}}{k+1}\left\{\sum_{n=1}^{\infty} \frac{\varphi_{r-1}(n)}{n^{k+2}}-\sum_{n=\left[\left(-\frac{m}{m}\right]+1\right.}^{\infty} \frac{\varphi_{r-1}(n)}{n^{k+2}}\right\}+0\left(m^{k+1 / 2}\right) \\
& =\alpha_{r, k} m^{k+1}+m^{(k+1) / 2}\left\{m^{(k+1) / 2} \sum_{n>\sqrt{m}} 0\left(\frac{1}{n^{k+1}}\right)\right\}+0\left(m^{k+1 / 2}\right) \\
& =\alpha_{r, k} m^{k+1}+0\left(m^{(k+2) / 2}\right)+0\left(m^{k+1 / 2}\right)=\alpha_{r, k} m^{k+1}+0\left(m^{k+1 / 2}\right) .
\end{aligned}
$$


If we substitute these estimates of $S_{1}, S_{2}$ and $S_{3}$ in (3.15) we get part b of Theorem 9 . The proof of part a is similar with the following changes. We have to replace $\varphi_{r-1}(n) / n$ by $\varphi_{r-1}^{*}\left(n, \beta_{r-1}(n)\right) / \beta_{r-1}(n)$ and use Corollary 2 instead of Corollary 1 to get an estimate similar to (3.14). The proof is complete.

We deduce a few corollaries to our theorem.

Corollary 5. If $\sigma(n)$ denotes the sum of the divisors of $n$ then

$$
\sum_{n=1}^{m} \sigma(n) \sim \frac{\pi^{2}}{12} m^{2}
$$

COROllary 6. If $\sigma_{r, k}(n)$ denotes the sum of the $k$ th powers of the divisors of $n$ then

$$
\sum_{n=1}^{m} \sigma_{1, k}(n)=\frac{\zeta(k+1)}{k+1} m^{k+1}+0\left(m^{k+1 / 2}\right)
$$

where $\zeta$ is Riemann's $\zeta$ function.

COROLlaRy 7. If $\sigma_{2,1}(n)$ denotes the sum of the unitary divisors of $n$ then

$$
\sum_{n=1}^{m} \sigma_{2,1}(n) \sim \frac{\pi^{2} m^{2}}{12 \zeta(3)}+0\left(m^{3 / 2}\right)
$$

Proofs. Corollary 5 follows from Theorem 9 if we estimate $\alpha_{11}$. Clearly

$$
\alpha_{11}=\alpha_{11}^{*}=\frac{1}{2} \sum_{n=1}^{\infty} \frac{1}{n^{2}}=\frac{\pi^{2}}{12} \text {. }
$$

Corollary 6 follows if we find $\alpha_{1, k}$ which is $\zeta(k+1) / k+1$. Corollary 7 comes out of an estimate of $\sigma_{2,1}$

$$
\sigma_{2,1}=\sigma_{2,1}^{*}=\frac{1}{2} \sum_{n=1}^{\infty} \frac{\varphi_{1}(n)}{n^{3}}=\frac{1}{2} \frac{\zeta(2)}{\zeta(3)}=\frac{\pi^{2}}{12 \zeta(3)}
$$

which is the result due to Cohen.

COROLlary 8 . For any $k \geqq 1$ we have

$$
\alpha_{2, k} \leqq \alpha_{4, k} \leqq \alpha_{6, k} \leqq \cdots \leqq \alpha_{5, k} \leqq \alpha_{3, k} \leqq \alpha_{1, k} .
$$

Also

$$
c_{1} \leqq c_{3} \leqq c_{5} \leqq \cdots \leqq c_{6} \leqq c_{4} \leqq c_{2} .
$$

These follow directly from Theorem 5 .

\section{Error functions}

We finally take up a discussion of error functions associated with the Euler functions. (A similar discussion for $r=1$ is made in Alladi (1974).) 
We first calculate the average value of $e_{r}(n, x)$ and $e_{r}^{*}(n, x)$ for fixed $n$ when $x$ is discrete.

THEOREM 10.

$$
\begin{aligned}
& \lim _{m \rightarrow \infty} \frac{1}{m} \sum_{i=1}^{m} e_{r}(n, i)=-\frac{\varphi_{r}(n)}{2 n} \\
& \lim _{m \rightarrow \infty} \frac{1}{m} \sum_{i=1}^{m} e_{r}^{*}(n, i)=\frac{-\varphi_{r}^{*}\left(n, \beta_{r}(n)\right)}{2 \beta_{r}(n)} .
\end{aligned}
$$

Proof. From Theorem 2 we deduce

$$
e_{r}(n, i)+e_{r}(n, n-i)=\left\{\begin{aligned}
0 & \text { if }(i, n)_{r} \neq 1 \\
-1 & \text { if }(i, n)_{r}=1
\end{aligned}\right.
$$

so that we get

$$
\sum_{i=1}^{n} e_{r}(n, i)=-\varphi_{r}(n) / 2
$$

Now Theorem 1 says

$$
\begin{aligned}
e_{r}(n, \lambda n+i) & =\frac{\lambda n+i}{n} \varphi_{r}(n, \lambda n+i)=\frac{\lambda n+i}{n} \varphi_{r}(n)-\lambda \varphi_{r}(n)-\varphi_{r}(n, i) \\
& =e_{r}(n, i) .
\end{aligned}
$$

Let $m=\lambda n+\mu$ for some non-negative $\lambda$, where $0 \leqq \mu<n$. Clearly

$$
\begin{aligned}
\frac{1}{m} \sum_{i=1}^{m} e(n, i)= & \frac{1}{m} \sum_{i=1}^{n} e_{r}(n, i)+\cdots \frac{1}{m} \sum_{i=(\lambda-1) n+1}^{\lambda n} e_{r}(n, i) \\
& +\frac{1}{m} \sum_{i=\lambda n+1}^{\lambda n+\mu} e_{r}(n, i) \\
= & \frac{-\lambda \varphi_{r}(n)}{2 m}+\frac{1}{m} \sum_{i=1}^{\mu} 0\left(n^{\xi}\right)=\frac{-\varphi_{r}(n)}{2 n}+0\left(\frac{1}{m}\right) .
\end{aligned}
$$

So that proceeding to the linit $m \rightarrow \infty$ we get the first part of the theorem The second part follows on similar reasoning.

However the mean over the continuous variable vanishes. To be more precise

$$
\int_{0}^{n} e_{r}(n, x)=0 ; \quad \int_{0}^{\beta_{r}(n)} e_{r}^{*}(n ; x) d x=0 .
$$

The above statement is an immediate consequence of the following statement: 
If $f$ is Riemann integrable in $[0, m]$ and $f(x)+f(m-x)=0$, for all but a finite number of $x \in[0, m]$ then $\int_{0}^{m} f(x) d x=0$. Clearly

$$
\int_{0}^{m} f(x) d x=\int_{0}^{m} f(m-x) d x=\frac{1}{2} \int_{0}^{1} f(x)+f(m-x) d x=0 .
$$

Note that $e_{r}(n, x)+e_{r}(n, n-x)=0$ for all $x$ except when $(x, n)_{r}=1$ and $e_{r}^{*}(n, x)+e_{r}^{*}\left(n, \beta_{r}(n)-x\right)=0$ except when $(n, x)_{r}=1$.

We now study the properties of the additive error functions associated with $\varphi_{r}$ and $\varphi_{r}^{*}$. Define for $s \geqq 2$

$$
e_{r}\left(n, \alpha_{1}, \alpha_{2}, \cdots, \alpha_{s}\right)=\varphi_{r}\left(n, \sum_{i=1}^{s} \alpha_{i}\right)-\sum_{i=1}^{s} \varphi_{r}\left(n, \alpha_{i}\right)
$$

and

$$
e_{r}^{*}\left(n, \alpha_{1}, \alpha_{2}, \cdots, \alpha_{s}\right)=\varphi_{r}^{*}\left(n, \sum_{i=1}^{s} \alpha_{i}\right)-\sum_{i=1}^{s} \varphi_{r}^{*}\left(n, \alpha_{i}\right) .
$$

We begin by showing

THEOREM 11.

(a)

$$
\begin{aligned}
\lim _{m \rightarrow x} \sum_{n=1}^{m} e_{r}\left(n, \alpha_{1}, \alpha_{2}, \cdots, \alpha_{s}\right)= & \sum_{n=1}^{\alpha_{1}+\alpha_{2}+\cdots+\alpha_{s}} \frac{\varphi_{r}^{*}\left(n, \beta_{r}(n)\right)}{\beta_{r}(n)} \\
& -\sum_{i=1}^{s} \sum_{n=1}^{\alpha_{i}} \frac{\varphi_{r}^{*}\left(n, \beta_{r}(n)\right)}{\beta_{r}(n)}
\end{aligned}
$$

and

(b) $\quad \lim _{m \rightarrow x} \frac{1}{m} \sum_{n=1}^{m} e_{r}^{*}\left(n, \alpha_{1}, \alpha_{2}, \cdots, \alpha_{s}\right)=\sum_{n=1}^{\alpha_{1}+\alpha_{2}+\cdots+\alpha_{s}} \frac{\varphi_{r}(n)}{n}-\sum_{i=1}^{s} \sum_{n=1}^{\alpha_{i}} \frac{\varphi_{r}(n)}{n}$.

Proof. We only prove the first part. The proof of (b) is similar. We know

(4.1) $\frac{1}{m} \sum_{n=1}^{m} e_{r}\left(n, \alpha_{1}, \alpha_{2}, \cdots, \alpha_{s}\right)=\frac{1}{m} \sum_{n=1}^{m} \varphi_{r}\left(n, \sum_{i=1}^{s} \alpha_{i}\right)-\frac{1}{m} \sum_{n=1}^{m} \sum_{i=1}^{s} \varphi_{r}\left(n, \alpha_{i}\right)$.

For any integer $j$ we have

$$
\begin{aligned}
\sum_{n=1}^{m} \varphi_{r}(n, j) & =\sum_{n=1}^{m} \sum_{\substack{i=1 \\
(i, n)_{r}=1}}^{j} 1=\sum_{n=1}^{j} \sum_{\substack{i=1 \\
(n,)_{r}=1}}^{m} 1=\sum_{n=1}^{j} \varphi_{r}^{*}(n, m) \\
& =m \sum_{n=1}^{j} \frac{\varphi_{r}^{*}\left(n, \beta_{r}(n)\right)}{\beta_{r}(n)}+0(1) .
\end{aligned}
$$

This implies that 


$$
\lim _{m \rightarrow \infty} \frac{1}{m} \sum_{n=1}^{m} \varphi_{r}(n, j)=\sum_{n=1}^{j} \frac{\varphi_{r}^{*}\left(n, \beta_{r}(n)\right)}{\beta_{r}(n)} .
$$

If in (4.3) we set $j$ as $\Sigma \alpha_{i}$, and as $\alpha_{i}$ and then use (4.1) and proceed to the limit $m \rightarrow \infty$ we get Theorem 11 part (a). Part (b) follows by observing that

$$
\lim _{m \rightarrow \infty} \frac{1}{m} \sum_{n=1}^{m} \varphi_{r}^{*}(n, j)=\sum_{n=1}^{j} \frac{\varphi_{r}(n)}{n} .
$$

Note that the right hand side of (b) and (a) are of the form

$$
g_{r}\left(\sum_{i=1}^{s} \alpha_{i}\right)-\sum_{i=1}^{s} g_{r}\left(\alpha_{i}\right)
$$

and

$$
g^{*}\left(\sum_{i=1}^{s} \alpha_{i}\right)-\sum_{i=1}^{s} g_{r}^{*}\left(\alpha_{i}\right)
$$

which resembles remarkably the forms of $e_{r}\left(n, \alpha_{1}, \alpha_{2}, \cdots, \alpha_{s}\right)$ and $e_{r}^{*}\left(n, \alpha_{1}, \alpha_{2}, \cdots, \alpha_{s}\right)$.

In fact as (4.3) and (4.4) are true the following can be shown without too much trouble.

$$
\lim _{m \rightarrow \infty} \frac{1}{m} \sum_{n=1}^{m} e_{r}(n, i)=2 c, i-\sum_{n=1}^{i} \frac{\varphi_{r}^{*}\left(n, \beta_{r}(n)\right)}{\beta_{r}(n)}=o(i)
$$

and

$$
\lim _{m \rightarrow \infty} \frac{1}{m} \sum_{n=1}^{m} e_{r}^{*}(n, i)=2 c_{r} i-\sum_{n=1}^{i} \frac{\varphi_{r}(n)}{n}=o(i)
$$

by virtue of Theorem 7. Compare (4.5) and (4.6) with Theorem 10.

We conclude by proving a necessary and sufficient condition for a number $n$ to be a power of a prime using $e_{r}\left(n, \alpha_{1}, \alpha_{2}\right)$.

THEOREM 12. A necessary and sufficient condition for $n$ to be a power of a prime is that

$$
e_{r}\left(n, \alpha_{1}, \alpha_{2}\right) \leqq 0 \forall \alpha_{1}, \alpha_{2} \in Z^{+}=\{1,2,3, \cdots\} .
$$

Proof. The necessity part is easy to establish. We know that

$$
\begin{gathered}
\varphi_{r}\left(n, \alpha_{1}+\alpha_{2}\right)=\alpha_{1}+\alpha_{2}-\left[\frac{\alpha_{1}}{p^{\frac{j_{r}(m)}{(m}}}\right] \\
\varphi_{r}\left(n, \alpha_{1}\right)=\alpha_{1}-\left[\frac{\alpha_{1}}{p^{f_{r}(m)}}\right] ; \quad \varphi_{r}\left(n, \alpha_{2}\right)=\alpha_{2}-\left[\frac{\alpha_{2}}{p^{f_{r}(m)}}\right]
\end{gathered}
$$

where $n=p^{m}, p$ prime. Now as $[x+y] \geqq[x]+[y]$ the necessity part follows directly. 
To prove sufficiency let (4.7) hold and let $n=\prod_{i=1}^{s} p_{i}^{\beta_{i}}, s>1$. We shall get a contradiction. Consider the two numbers $p_{i}^{f_{i}\left(\beta_{i}\right)}$ and $p_{i}^{f_{r}\left(\beta_{i}\right)}$ for distinct $i, j$ with $1 \leqq i<j \leqq s$. As these numbers are relatively prime, there exist integers $x$ and $y$ positive so that

$$
\left|x p_{i}^{f_{r}^{(}\left(\beta_{i}\right)}-y p_{j}^{f_{r}\left(\beta_{j}\right)}\right|=1 .
$$

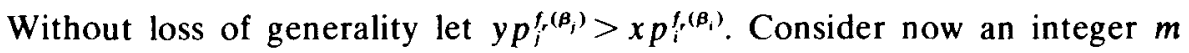
satisfying

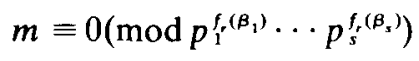

and let

$$
m^{\prime}=\prod_{i=1}^{s} p_{i}^{f_{i}^{\left(\beta_{i}\right)} .}
$$

One can show that $(a, n)_{r}=1$ if and only if

$$
\left(\lambda m^{\prime}+a, n\right)_{r}=\left(\lambda m^{\prime}-a, n\right)_{r}=1 .
$$

Now consider the intervals $\left(0, y p_{i}^{f_{i}\left(\beta_{i}\right)}\right]$ and $\left(m-2, m+y p_{j}^{f_{i}\left(\beta_{i}\right)}-2\right]$. It is evident from (4.11) that for every $a$ with $0<a \leqq y p_{i}^{f_{r}\left(\beta_{j}\right)}-2$ and $(a, n)_{r}=1$ we have equivalently an $m+a$ satisfying $m<m+a \leqq m+y p_{j}^{f_{r}\left(\beta_{j}\right)}-2$ and

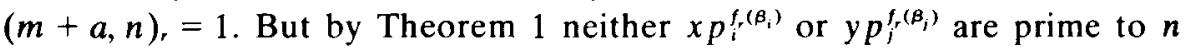
order $r$. Yet as $(1, n)_{r}=1$ we have $(m-1, n)_{r}=1$. Thus

$$
\varphi_{r}\left(n, m-2, m+y p_{i}^{f_{r}\left(\beta_{i}\right)}-2\right)=\varphi_{r}\left(n, y p_{i}^{f_{r}\left(\beta_{i}\right)}\right)+1
$$

which is the same as saying

$$
e_{r}\left(n, \alpha_{1}, \alpha_{2}\right)=1>0
$$

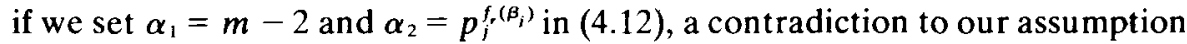
(4.7). Thus $s=1$ which establishes sufficiency. The proof is complete.

\section{Acknowledgement}

I am thankful to the referee for his comments and suggestions.

I also thank Professors E. G. Straus, H. G. Niederreiter and Ian Richards for their criticisms and for stimulating discussions.

\section{References}

K. Alladi (1974), On a generalisation of the Euler function (Srinivasa Ramanujan Commemoration Volume, Oxford Press, Madras, India, 114-124).

J. Chidambaraswamy (1967), 'Sum functions of unitary and semi unitary divisors', J. Indian. Math. Soc. 31, 117-126. 
E. Cohen (1960), 'Arithmetic functions associated with the unitary divisors of an integer', Math. Z. 74, 66-80.

W. J. LeVeque (1960), Topics in Number Theory (Vol. 1, Addison Wesley, Reading, Mass. 103-114).

D. Suryanarayana (1971), 'On the number of bi-unitary divisors of an integer', Proc. Conf. on Arithmetic Functions, Springer-Verlag 251, 272-282.

Department of Mathematics,

Vivekananda College,

Madras, India.

Present address:

Department of Mathematics,

University of California,

Los Angeles, U.S.A. 